\title{
Bulk tank milk antibody ELISA as a biosecurity tool for detecting dairy herds with past exposure to Mycoplasma bovis
}

\author{
A. M. Parker, ${ }^{* 1}$ J. K. House, ${ }^{*}$ M. S. Hazelton, ${ }^{*}$ K. L. Bosward, ${ }^{*}$ J. M. Morton, $\dagger$ and P. A. Sheehy ${ }^{*}$ \\ *Faculty of Science, Sydney School of Veterinary Science, The University of Sydney, Camden, New South Wales 2570, Australia \\ †Jemora Pty Ltd., Geelong, Victoria 3220, Australia
}

\begin{abstract}
In Australia, one of the biosecurity recommendations to help prevent the introduction of Mycoplasma bovis into a dairy herd is to use a PCR assay on bulk tank milk (BTM) samples to evaluate the M. bovis infection status of potential source herds. An alternative approach is to assess the immunological status of the herd with respect to previous exposure to $M$. bovis via the use of an ELISA that is commercially available for use on cattle milk and serum. The objectives of this study were to (1) evaluate factors potentially associated with variation in the ELISA BTM optical density coefficient (ODC\%) in previously exposed herds, (2) evaluate the association between the proportion of cows that are ELISA positive and the BTM ELISA ODC\%, (3) assess agreement between the BTM ELISA and PCR and culture, and (4) compare BTM ELISA ODC\% between the "hospital" herd and the main lactating herd on the same farm. Bulk tank milk samples $(\mathrm{n}=192)$ were collected from 19 dairy herds with a history of clinical $M$. bovis disease and from 6 control herds (herds with no known clinical cases of mycoplasmosis). For 28 of the BTM samples collected, blood was also collected from 50 lactating cows contributing to that bulk tank sample. From 1 herd, concurrent paired BTM samples were collected from the main herd and the hospital herd on 16 occasions. All BTM samples were analyzed by ELISA (Bio-X Bio K 302, Bio-X Diagnostics, Rochefort, Belgium), PCR, and culture. The BTM ELISA ODC\% was associated with time since initial $M$. bovis outbreak and time since the start of the herd's calving period. Following an initial outbreak of $M$. bovis, the BTM ELISA ODC\% was highest in the first 8 mo. In split- and seasonal-calving herds, significantly higher BTM ELISA ODC\% results were observed 5 to $8 \mathrm{wk}$
\end{abstract}

Received December 15, 2016.

Accepted June 21, 2017.

${ }^{1}$ Corresponding author: alysia.parker@sydney.edu.au after the commencement of the calving period. A significant association was observed between the within-herd seroprevalence for the lactating herd and BTM ELISA ODC\%, but within-herd seroprevalence explained little of the variation in BTM ELISA ODC\%. When comparing the BTM ELISA with a multiplex probe PCR and culture followed by $16 \mathrm{~S}$ to $23 \mathrm{~S}$ rRNA sequencing, there was virtually no agreement above that expected by chance; prevalence-adjusted bias-adjusted kappa values were 0.22 and 0.25 for ELISA category versus PCR category and culture, respectively. Finally, the hospital herd BTM ELISA ODC\% mirrored that for the main herd BTM but was significantly higher. This study demonstrates that this commercially available ELISA used on BTM samples may complement the use of BTM PCR or culture in identifying herds from which purchase of animals may pose a higher biosecurity risk for introduction of $M$. bovis into noninfected herds.

Key words: enzyme-linked immunosorbent assay, Mycoplasma bovis, bulk tank milk, antibody

\section{INTRODUCTION}

Mycoplasma bovis is one of the most pathogenic species of Mycoplasma in cattle, causing significant economic losses in infected herds worldwide (Nicholas, 2011). In adults, clinical signs are predominantly associated with mastitis and arthritis, whereas in young calves signs include otitis media, arthritis, and pneumonia (Maunsell et al., 2011). Mycoplasma bovis was first isolated in Australia in 1970 (Cottew, 1970). However, in recent years there has been an increase in the number of outbreaks detected within the Australian dairy industry, and strategies are needed to curb the spread of this pathogen throughout the industry. Mycoplasma bovis is a highly contagious pathogen that is refractory to antimicrobials registered for treatment of lactating dairy cattle (Dairy Australia Limited, 2014). This, in conjunction with the role of subclinical carrier animals, makes elimination of the pathogen from a herd very difficult (Maunsell et al., 2011). As a result, a large focus should be placed on biosecurity measures to prevent 
the introduction of $M$. bovis into a herd and on controlling clinical M. bovis disease if a herd becomes infected.

Collecting bulk tank milk (BTM) samples is quick and simple, and such samples provide a good representation of the lactating herd. Therefore, one of the current biosecurity recommendations in Australia to reduce the risk of introduction of $M$. bovis into a naïve dairy herd is to assess the BTM of the main herd and the "hospital" herd from which the purchase of animals is being considered using PCR or culture (Dairy Australia Limited, 2014). Both PCR and culture have been used on BTM samples as surveillance tools to identify the herd-level prevalence of Mycoplasma species across different regions and countries (Higuchi et al., 2011; Justice-Allen et al., 2011). However, this approach has limitations. To obtain a positive result, infected cows in the herd must be contributing to the bulk tank and must be shedding the pathogen at the time the BTM sample is collected. Mycoplasma shedding is known to be intermittent and inconsistent in cows with IMI (Biddle et al., 2003), and milk from clinically affected cows is not routinely collected into the bulk tank because clinically affected cows would usually be moved to the hospital herd. Thus, there is potential to miss identifying infected herds when relying on culture and PCR of BTM samples alone.

A commercially available antibody ELISA kit (BioX Bio K 302, Bio-X Diagnostics, Rochefort, Belgium) has been designed to detect specific antibodies against $M$. bovis in either milk or serum as an indicator of past exposure. Shedding of the pathogen at the time of BTM sample collection is not required for disease detection as for PCR or culture. Recent studies have evaluated the use of this kit for BTM and serum sample analysis. Using latent class analysis, the first study evaluated the performance of the ELISA as a herdlevel diagnostic tool on BTM as compared with PCR for detecting infected herds; medians of their estimated herd-level sensitivity and specificity distributions were $60.4 \%$ (95\% probability interval: $37.5-96.2$ ) and $97.3 \%$ (95\% probability interval: 94.0-99.8), respectively, when using the manufacturer's recommended cutoff optical density coefficient (ODC\%) value of 37 (Nielsen et al., 2015). A separate study evaluated the use of the ELISA on serum samples from individual calves experimentally exposed to aerosols containing $M$. bovis. Relative sensitivity and specificity compared with an M. bovis-specific IgG ELISA were 37\% (95\% CI: 22-54) and 95\% (95\% CI: 83-99), respectively (Wawegama et al., 2016). A third study investigated factors that may affect the BTM ELISA ODC\%; a significant positive relationship was observed between the within-herd ELISA apparent prevalence in lactating cows (based on individual composite milk samples) and the BTM
ELISA ODC\% (Petersen et al., 2016). Neither withinherd seroprevalence in young stock nor herd size was associated with BTM ELISA ODC\%. Due to the limited published literature on this ELISA and evaluation of its use in Australian dairy production systems, additional research is required to evaluate further factors that may affect the BTM ELISA ODC\%. This will assist understanding of its usefulness as a biosecurity tool for identifying herds that have been exposed to $M$. bovis and pose a biosecurity risk to unexposed herds planning to purchase and introduce new stock. The objectives of this study were to (1) evaluate factors potentially associated with variations in the ELISA BTM ODC\% in previously exposed herds, (2) evaluate the association between the proportion of cows that are ELISA positive and the BTM ELISA ODC\%, (3) assess the use of the BTM ELISA as a diagnostic tool when compared with PCR and culture by assessing agreement between these tests, and (4) compare BTM ELISA ODC\% between the hospital herd and the main lactating herd on the same farm.

\section{MATERIALS AND METHODS}

\section{Herd Selection}

The study population consisted of herds chosen from the client base of The University of Sydney Livestock Veterinary Teaching and Research Unit (LVTRU; Camden, New South Wales, Australia) in their capacity of providing veterinary services for clinical disease management. From those dairy herds with a history of clinical $M$. bovis disease diagnosed by culture or PCR within the previous $2 \mathrm{yr}$, a convenience sample of 19 previously exposed herds was selected. The University of Sydney LVTRU had assisted with managing the $M$. bovis disease within these herds (Supplemental Table S1; https://doi.org/10.3168/jds.2016-12468). For each herd, the first BTM sample for the current study was collected less than 24 mo after clinical $M$. bovis disease was evident in the herd. The BTM samples contained 1 or 2 milking sessions as milk was picked up on a daily basis from all herds except herd 11, in which milking occurred continuously; most cows were milked 3 times daily, and 3 bulk tanks were used and emptied several times throughout the day. A convenience sample of 6 dairy herds with no known clinical cases of mycoplasmosis was also selected as a control group (Supplemental Table S1). These herds were selected because of their long-standing close association with the University of Sydney LVTRU. Our familiarity with these herds' long-term disease histories provided high confidence that none had experienced clinical signs of $M$. bovis disease either within the last $5 \mathrm{yr}$ of the first 
BTM sample collected for inclusion in this study or during the study period. Of the 6 control herds, 3 herds (herds 20, 21, and 23; Supplemental Table S1) were extensively examined for the presence of $M$. bovis. From each of these herds, nose swabs, eye swabs, vaginal swabs, and composite milk samples were collected from 50 systematically selected cows (e.g., every second cow for a herd of 100) contributing to the main BTM on the day of sampling. All samples were culture negative for M. bovis.

The 19 herds with a history of $M$. bovis were located in the states of Tasmania $(\mathrm{n}=14)$, New South Wales $(\mathrm{n}=2)$, Victoria $(\mathrm{n}=2)$, and South Australia $(\mathrm{n}=1)$. All control herds were located in New South Wales (n $=6$ ). In regards to calving system, 11 of the 19 previously exposed herds calved seasonally (i.e., each year all cows calved within a single restricted time period) in autumn (March through May; $\mathrm{n}=4$ ) or spring (September through November; $\mathrm{n}=7$ ); 5 used split calving, with all cows calving in 2 restricted periods each year in autumn (March through May) and spring (September through November); and 2 herds used year-round calving. Calving system was not recorded for 1 herd (Table S1). The 6 control herds used year-round calving.

\section{Sample Analyses}

For the detection of $M$. bovis antibodies in BTM and individual animal serum samples, the Bio-X Bio K 302 Mycoplasma bovis ELISA kit was used (Bio-X Diagnostics, Rochefort, Belgium) according to the manufacturer's instructions. In brief, serum samples and the supplied positive and negative controls were diluted $1 / 100$ in the supplied dilution buffer. Milk samples were centrifuged for $20 \mathrm{~min}$ at $4,000 \times \mathrm{g}$. The upper layer of fat was removed, and the undiluted skim milk samples were used in the wells. Samples were added in duplicate $100-\mu \mathrm{L}$ volumes to the wells of the supplied ELISA plates. On each plate, blank wells (dilution buffer) were included in duplicate, and positive and negative controls were added in triplicate $100-\mu \mathrm{L}$ volumes. Plates were incubated with a lid for $1 \mathrm{~h}$ at 21 $\pm 3^{\circ} \mathrm{C}$. Plates were washed 3 times with the supplied wash solution. The supplied conjugate was diluted 1:50 with the supplied dilution buffer, and $100 \mu \mathrm{L}$ was added to each well followed by incubation with a lid for $1 \mathrm{~h}$ at $23 \pm 3^{\circ} \mathrm{C}$. Plates were washed as described previously. The supplied chromogen solution was then added to each well in 100- $\mu \mathrm{L}$ volumes and incubated for $10 \mathrm{~min}$ at $21 \pm 3^{\circ} \mathrm{C}$ uncovered and protected from the light; then, the supplied stop solution was added to each well in $50-\mu \mathrm{L}$ volumes. The optical density (OD) of each well was then read immediately at $450 \mathrm{~nm}$ on a microplate reader (Fluostar Optima, BMG LabTech,
Ortenberg, Germany). Sample coefficients were calculated per the manufacturer's instructions: ODC\% $=(\mathrm{OD}$ sample $-\mathrm{OD}$ negative control $) /(\mathrm{OD}$ positive control - OD negative control) $\times 100$. Each ELISA plate was validated using the specified OD threshold for the positive and negative controls. The coefficient of variation was calculated for each sample. Samples with an OCD $\%>20$ and a coefficient of variation $>0.2$ were reanalyzed. Per the manufacturer's recommendations, a sample coefficient $\geq 37$ ODC $\%$ was considered positive and a sample coefficient $<37$ ODC\% was considered negative for both BTM and individual serum samples.

All cultures were performed on Mycoplasma agar [Mycoplasma agar base (CM0401, Oxoid, Hampshire, UK); distilled water; $0.2 \%$ (wt/vol) calf thymus DNA (D1501, Sigma, St. Louis, MO); and Mycoplasma Selective Supplement G (SR0059C, Oxoid)] and incubated at $37^{\circ} \mathrm{C}$ in candle jars for elevated $\mathrm{CO}_{2}$ levels for 5 to $10 \mathrm{~d}$. Colonies with morphology consistent with mycoplasma were placed in PBS for speciation by an in house-developed PCR assay targeting the $16 \mathrm{~S}$ to 23S rRNA intergenic spacer region of Mycoplasma spp. as described by Parker et al. (2017). The PCR products from this assay were then speciated via Sanger Sequencing (Australian Genome Research Facility Ltd., Sydney, New South Wales, Australia).

For PCR assays, DNA extractions were performed on approximately $1 \mathrm{~mL}$ of milk and centrifuged at 13,000 $\times g$ for 5 min (Eppendorf Centrifuge 5424, Eppendorf South Pacific Pty. Ltd. North Ryde, New South Wales, Australia); this was followed by the removal of fat and supernatant. The remaining pellet was resuspended in $90 \mu \mathrm{L}$ of Buffer ATL (Qiagen Pty Ltd., Chadstone Centre, Victoria, Australia) and $10 \mu \mathrm{L}$ of Proteinase $\mathrm{K}$ (Qiagen) and incubated at $56^{\circ} \mathrm{C}$ for 1 to $3 \mathrm{~h}$ with occasional vortexing. The DNA extraction process was then continued using the BioSprint 96 One-For-All Vet kit (Qiagen) following the protocol for purification of viral nucleic acids and bacterial DNA from animal tissue homogenates, serum, plasma, other body fluids, swabs, and washes. All buffers and plasticware used were supplied by the kit. Briefly, $40 \mu \mathrm{L}$ of proteinase $\mathrm{K}$ was added to the bottom of each well of a 96 -well S-Block. Prepared samples were then added to the wells in $100-\mu \mathrm{L}$ volumes. A Buffer RLT mixture was prepared containing Buffer RLT (35 mL), isopropanol (35 mL), MagAttract suspension $\mathrm{G}(3 \mathrm{~mL})$, and carrier RNA $(1 \mu \mathrm{g} / \mu \mathrm{L}, 310 \mu \mathrm{L})$ and was added to each well in $600-\mu \mathrm{L}$ volumes. One additional 96-well S-Block was prepared containing Buffer AW1 (700 $\mu \mathrm{L} /$ well), and 2 additional 96-well S-Blocks containing Buffer RPE $(500 \mu \mathrm{L} /$ well) were prepared. Two 96-well microplates were prepared, with one containing elution Buffer AVE (75 $\mathrm{LL} /$ well) and the other containing a 96-Rod Cover. 
Extraction plates were then run on the BioSprint 96 (Qiagen) using the BS96 Vet 100 protocol according to the Biosprint 96 One-For-All Vet Handbook (Qiagen). Each extraction plate included a blank extraction control that was analyzed by PCR alongside the milk samples. The PCR assay used was a Mycoplasma species-specific real-time probe-based multiplex PCR assay as described by Parker et al. (2017) targeting M. bovis, Mycoplasma californicum, and Mycoplasma bovigenitalium. To validate the DNA extraction process, an in house-developed control PCR assay targeting the Bos taurus mitochondrial cytochrome $\mathrm{b}$ gene was also used on all samples as described by Parker et al. (2017). All samples were analyzed in triplicate for the Mycoplasma assay and in singletons for the control mitochondrial cytochrome b assay and were considered positive if a cycle threshold $<40$ was achieved.

\section{Factors Potentially Associated with BTM ELISA ODC\% in Previously Exposed Herds}

For factors potentially associated with BTM ELISA ODC\% in previously exposed herds, BTM samples (n $=186$ ) were collected from the 19 previously exposed study herds (Supplemental Figure S1; https://doi.org/ 10.3168/jds.2016-12468), including 13 herds in which ongoing BTM samples were collected on a fortnightly to monthly basis when possible (Supplemental Table S1). During the sampling period, close communication was maintained with each farm and all $M$. bovis test results were recorded, including those from individual animals and BTM samples. Calving dates for cows in each herd were also obtained with the exception of 1 herd. The following potential explanatory factors for BTM ELISA ODC\% were analyzed.

- Herd size: continuous data; defined for year-roundcalving herds as the average number of cows in the milking herd during the study period and for seasonal- and split-calving herds as the approximate number of cows calved in the 12-mo period.

- Bulk tank milk $M$. bovis PCR result from the same BTM sample: binary data, where all BTM samples were analyzed by PCR and each result was classified as either positive or negative.

- Bulk tank milk $M$. bovis culture result from the same BTM sample: binary data, where all BTM samples were analyzed by culture and each result was classified as either positive or negative.

- Time since the start of the calving period: categorical data, where each BTM sample was categorized based on time from the beginning of the most recent calving period to BTM sampling date. Categories were year-round calving and, for seasonal- and split-calving herds, 1 to $4 \mathrm{wk}, 5$ to $8 \mathrm{wk}, 9$ to $12 \mathrm{wk}, 13$ to $20 \mathrm{wk}, 21$ to $28 \mathrm{wk}$, and $>28$ wk.

- Time since most recent M. bovis positive culture or PCR: categorical data, where each BTM sample was categorized based on time from the herd's most recent positive M. bovis test to BTM sampling date. Positive $M$. bovis tests were any recorded positive culture or PCR tests on samples from individual animals and BTM samples collected by the herd veterinarian or herd manager. Categories were 0 to 3,4 to 6,7 to 12,13 to 18,19 to 30,31 to 42,43 to 54 , and $>54 \mathrm{wk}$.

- Time since initial M. bovis outbreak: categorical data, where each BTM sample was categorized based on time from the initial $M$. bovis outbreak of clinical disease of that herd to BTM sampling date. An outbreak was defined as a herd experiencing 1 or more cases of clinical disease caused by $M$. bovis as confirmed by culture or PCR. The categories were 0 to 4,5 to 8,9 to 12,13 to 16,17 to 20,21 to 24 , and $>24$ mo.

\section{Association Between the Proportion of Cows that Were ELISA Positive and the BTM ELISA ODC\%}

A total of 23 of the 25 study herds were included in this analysis (17 previously exposed herds and 6 control herds; Supplemental Figure S1); 3 of those herds were sampled twice on separate occasions and 1 herd was sampled 3 times on separate occasions. All other herds were sampled once. This gave a total of 28 samplings. For each sampling, a BTM sample was collected, and blood samples were collected from 50 systematically selected cows (e.g., every second cow for a herd of 100) that were contributing to the main BTM on the day of sampling. For the 3 control herds that were extensively examined for the presence of $M$. bovis as previously described, the same 50 animals were used for blood collection. The sample size of 50 cows was chosen for both statistical and practical reasons, allowing reasonable precision but also being acceptable to herd managers and staff. At an observed seroprevalence of $20 \%$, precision (assessed as half of the exact mid- $P$ 95\% confidence interval) was approximately $\pm 10 \%$. Blood samples were collected during or immediately following a milking session. The BTM sample was collected from the main BTM at the end of that milking session and as such contained 1 or 2 milking sessions as the bulk tanks were emptied once daily. This was with the exception of herd 11, where milking occurred continuously. Most cows were milked 3 times daily; 3 bulk tanks were used, and these tanks were emptied several times throughout the day. In this herd, each BTM sample was from 
milk from a subset of all lactating cows being milked in the herd at that time. The within-herd seroprevalence of $M$. bovis antibody-positive lactating cows from the main milking herd was calculated as the proportion of the 50 sampled cows whose individual ELISA sample coefficient (ODC\%) value was $\geq 37$ per the manufacturer's directions for individual animal testing and as previously described (Petersen et al., 2016).

\section{Agreement Between ELISA Versus PCR and Culture}

Using the manufacturer's recommended cutoff of 37 ODC\% (i.e., $\geq 37$ was classified as positive) for individual animal testing, the ELISA value for each BTM sample $(\mathrm{n}=192$ from the 19 previously exposed herds and 6 control herds; Supplemental Figure S1) was classified as either positive or negative. These were then used to assess agreement between BTM ELISA category and each of the conventional tests of PCR and culture using prevalence-adjusted bias-adjusted kappa (PABAK) values as described in the Statistical Analyses section.

\section{Main BTM Versus Hospital BTM ELISA ODC\%}

Concurrent paired BTM samples were collected from the main herd and the hospital herd from a single splitcalving study herd of approximately 2,000 lactating cows (herd 5; Supplemental Table S1). The hospital herd contained lactating cows isolated from the main herd due to sickness, recent parturition, or antimicrobial residues. Their milk was collected separately from the main herd's bulk milk tank. Samples were collected on 16 occasions on a fortnightly to monthly basis for 1 yr (Feb. 1, 2015, to Jan. 31, 2016). This first sample was collected approximatly19 mo after the herd's initial outbreak and approximately 10 mo after the herd's most recent $M$. bovis positive culture or PCR of an individual animal or BTM. During the sampling period, no known clinical cases of $M$. bovis, as diagnosed by PCR or culture, were detected. All BTM samples from the main herd $(\mathrm{n}=16)$ and the hospital herd $(\mathrm{n}=16)$ were analyzed by PCR and culture, and a single BTM sample from the main herd was positive by PCR (Oct. $12,2015)$.

\section{Statistical Analyses}

To assess whether BTM ELISA ODC\% were continuous data, a 10-fold dilution series on 3 serum samples (very high ODC\%, high ODC\%, and medium ODC\%) and 3 milk samples (very high ODC\%, high ODC\%, and medium ODC\%) was performed using the supplied dilution buffer and analyzed by ELISA. This was followed by a 2-fold serial dilution series on 5 serum samples (starting value of approximately 100 ODC\%) and 4 milk samples (starting value of approximately 100 ODC\%) analyzed by ELISA. These results demonstrated that the ODC\% increased approximately linearly with concentration, indicating that it adequately met the assumption that it was continuous (interval) data for statistical purposes.

For all statistical analyses, statistical significance was declared at $P<0.05$. An Anderson-Darling test for normality was first performed on the BTM ELISA ODC\% data; values were $\log _{\mathrm{e}}$ transformed to fulfill normality requirements. Explanatory variables considered for analysis of factors affecting BTM ELISA ODC\% were herd size, BTM $M$. bovis PCR result, BTM M. bovis culture result, time since the start of the calving season, time since the most recent $M$. bovis positive test, and time since initial $M$. bovis outbreak. Herd of origin and sample collection date were included as random effects. A first-order autoregressive residual correlation structure was used to account for correlation between herd samplings within the same herd. The herd sampling was the unit of analysis where a BTM result in 1 herd constituted 1 herd sampling. A univariable linear mixed REML model was first constructed (GenStat 16th ed.; VSN International, Hemel Hempstead, UK) for each explanatory variable. Significant variables $(P<0.05$ on univariable analysis) were then included in a multivariable linear mixed REML model. A backward elimination approach was used to determine the final model in which all explanatory variables were significant $(P<$ 0.05 ), and 2-way interactions were investigated. Once eliminated, variables were not eligible for re-entry. Correlations between the final explanatory variables were assessed using Spearman's rank correlation coefficient. To assess for confounding, effect estimates were compared with and without potential confounding variables fitted. Model-based means were calculated as fitted means on the $\log _{\mathrm{e}}$ scale with random effects for herd of origin and sample collection date set to zero. These means were then back-transformed and therefore were geometric means. Effect estimates were exponentiated to provide estimated ratios of geometric means.

For analysis of the association between withinherd (i.e., cow level) seroprevalence and BTM ELISA ODC\%, the BTM ELISA ODC values were plotted against the within-herd seroprevalence. A linear mixed REML analysis was performed (GenStat 16th ed.) using $\log _{\mathrm{e}}$ transformed BTM ELISA ODC\% as the dependent variable, herd seroprevalence and herd size as the explanatory variables, and herd of origin fitted as a random effect. A variable was eliminated from the model if statistical significance was not achieved (i.e., if $P>0.05)$. 
Table 1. Descriptive statistics for analysis of factors affecting bulk tank milk (BTM) ELISA optical density coefficient (ODC\%) in 19 previously exposed herds (185 samples)

\begin{tabular}{lccc}
\hline Item & Minimum & Median & Maximum \\
\hline Herd size $^{1}$ & 340 & 840 & 3,000 \\
No. of samples/herd & 1 & 11 & 32 \\
BTM ELISA ODC\% & 10 & 33 & 179 \\
\hline
\end{tabular}

${ }^{1}$ In Australia in 2016, the average dairy herd size was 284 cows (Dairy Australia Limited, 2016).

Due to the possibility that BTM samples from herd 11 contained an inconsistent number of animals, analysis of factors affecting BTM ELISA ODC\% and analysis of the association between within-herd seroprevalence and BTM ELISA ODC\% was also performed with these samples excluded. Similar results were achieved; therefore, these samples were included in the final analyses.

For comparison of diagnostic tools, 2-sided McNemar's tests were performed (GenStat 16th ed.), and PABAK values were calculated separately for BTM ELISA category and PCR and for BTM ELISA category and culture. Clustering of samples within herds was disregarded; therefore, the $P$-values and $95 \%$ confidence intervals may have been spuriously smaller and narrower, respectively, than if there had been no clustering. For comparison of mean BTM ELISA ODC\% values from the main herd and the hospital herd, a paired 2-sample $t$-test was performed using $\log _{\mathrm{e}}$ transformed data (GenStat 16th ed.).

\section{RESULTS}

\section{Factors Affecting Variation in BTM ELISA ODC\%}

The final multivariable model contained only the 2 variables with significant effects when adjusted for the other and included 185 observations from 18 of the 19 previously exposed study herds; 1 observation and herd was excluded due to missing data for time since the beginning of the calving period. Descriptive statistics of herd size, samples used per herd, and the BTM ELISA ODC\% are shown in Table 1 . The $P$-values for the univariable models, multivariable model (i.e., the model with the 2-way interaction term included), and final multivariable model (interaction term removed) are shown in Table 2. The final multivariable model included 2 explanatory variables: time since initial $M$. bovis outbreak $(P<0.001)$ and time since the start of the calving season $(P<0.001)$. These 2 explanatory variables were weakly positively correlated $(\mathrm{r}=0.31)$. Effect estimates are shown in Table 3. The unadjusted effect estimate for year-round calving was confounded by time since initial $M$. bovis outbreak; the estimated ratio increased from 0.86 before adjustment to $1.41 \mathrm{af}-$ ter adjustment. Changes for other effect estimates were smaller.

The model-based means for time since initial $M$. bovis outbreak are shown in Figure 1. The model-based mean BTM ELISA ODC\% started above the positive-negative cutoff of $37 \mathrm{ODC} \%$ at 0 to 4 mo with a model-based mean ODC\% of 50, varying from 20 to 77 for individual herds. This was significantly higher than all other time since outbreak categories with the exception of 5 to 8 mo, where the model-based mean ODC\% peaked at 59 (varying from 28 to 91 for individual herds). A gradual decline in means was then observed to $>24$ mo with a model-based mean ODC\% of 20 (varying from 12 to 40 for individual herds), which was significantly lower than all other time since outbreak categories with the exception of 13 to 16 mo.

The model-based means for time since the start of the calving period are shown in Figure 2. Year-round calving had the highest model-based mean BTM ELISA ODC\%, with a model-based mean ODC\% (minimum, maximum) of 46 (varying from 16 to 51 for individual herds), and was significantly higher than seasonal- and split-calving herd categories of 13 to $20 \mathrm{wk}$ and later. For seasonal- and split-calving herds, at 1 to 4 wk the model-based mean ODC\% was 33 (varying from 17 to 43 for individual herds) before peaking at 5 to $8 \mathrm{wk}$

Table 2. $P$-values for the univariable models, multivariable model with interaction term, and final multivariable model for the analysis of factors affecting bulk tank milk (BTM) ELISA optical density coefficient

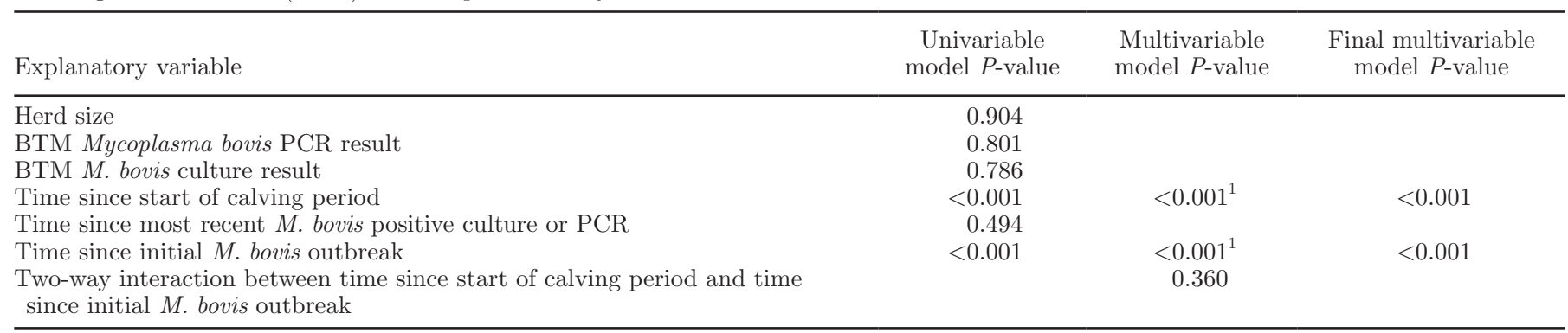

${ }^{1} P$-value for this explanatory variable at the reference category of the other explanatory variable. 
Table 3. Back-transformed variances and effect estimates (estimated ratios of geometric means) for the final model of bulk tank milk ELISA optical density coefficient

\begin{tabular}{|c|c|c|c|}
\hline Explanatory variable & Variance $^{1}$ & $\begin{array}{l}\text { Estimated ratio } \\
\text { of geometric means }{ }^{2}\end{array}$ & $\mathrm{SE}^{3}$ \\
\hline \multicolumn{4}{|l|}{ Random effects } \\
\hline Herd of origin & 1.05 & & 0.03 \\
\hline Sample collection date & 1.82 & & 0.51 \\
\hline Residuals & 1.07 & & 0.01 \\
\hline \multicolumn{4}{|l|}{ Fixed effects } \\
\hline Constant & & $48.67^{4}$ & 7.87 \\
\hline \multicolumn{4}{|c|}{ Time since start of calving period (wk) } \\
\hline Year round & & 1.41 & 0.33 \\
\hline $1-4^{5}$ & & - & - \\
\hline $5-8$ & & 1.27 & 0.16 \\
\hline $9-12$ & & 1.11 & 0.14 \\
\hline $13-20$ & & 0.96 & 0.13 \\
\hline $21-28$ & & 0.76 & 0.11 \\
\hline$>28$ & & 0.80 & 0.10 \\
\hline \multicolumn{4}{|c|}{ Time since initial Mycoplasma bovis outbreak (mo) } \\
\hline $0-4^{5}$ & & - & - \\
\hline $5-8$ & & 1.19 & 0.14 \\
\hline $9-12$ & & 0.74 & 0.10 \\
\hline $13-16$ & & 0.53 & 0.10 \\
\hline $17-20$ & & 0.54 & 0.07 \\
\hline $21-24$ & & 0.59 & 0.08 \\
\hline$>24$ & & 0.40 & 0.06 \\
\hline \multicolumn{4}{|c|}{${ }^{1}$ Variances are on the back-transformed scale, calculated as the $\log _{\mathrm{e}}$ transformed value exponentiated. } \\
\hline \multicolumn{4}{|c|}{$\begin{array}{l}{ }^{2} \text { Effect estimates were exponentiated (back-transformed) to provide estimated ratios of geometric means for } \\
\text { each category relative to the reference category. }\end{array}$} \\
\hline \multicolumn{4}{|c|}{$\begin{array}{l}{ }^{3} \text { Standard errors are on the back-transformed scale, calculated as the SE on the } \log _{\mathrm{e}} \text { transformed scale multi- } \\
\text { plied by the relevant back-transformed estimate or variance. }\end{array}$} \\
\hline \multicolumn{4}{|c|}{$\begin{array}{l}{ }^{4} \text { Fitted bulk tank milk ELISA optical density coefficient value for a seasonal- or split-calving herd } 1 \text { to } 4 \mathrm{wk} \\
\text { after the start of the calving period and } 0 \text { to } 4 \text { mo after the herd's initial } M \text {. bovis outbreak, with random effects } \\
\text { for herd of origin and sample collection date set to zero and then back-transformed. }\end{array}$} \\
\hline
\end{tabular}

with a model-based mean ODC\% of 41 (varying from 19 to 64 for individual herds). This was significantly higher than all other seasonal- and split-calving herd categories with the exception of 1 to 4 wk and 9 to 12 wk. There was then a gradual decline to $>28 \mathrm{wk}$, with a model-based mean ODC $\%$ of 26 (varying from 9 to 56 for individual herds). The model-based mean BTM ELISA ODC\% was above the positive-negative cutoff of $37 \mathrm{ODC} \%$ for year-round-calving herds and at 5 to 8 wk for seasonal- and split-calving herds.

\section{Association Between the Proportion of Cows that Were ELISA Positive and the BTM ELISA ODC\%}

The model included 28 samplings from 23 herds. Descriptive statistics of herd size, within-herd seroprevalence, and BTM ELISA ODC\% are shown in Table 4. The association between within-herd seroprevalence and BTM ELISA ODC\% is shown in Figure 3. Bulk tank milk ELISA ODC\% was significantly associated with within-herd seroprevalence $(P=0.027$; estimated increase in BTM ELISA ODC\% per $10 \%$ increase in within-herd seroprevalence was 1.14; 95\% CI: $1.02-$
1.27). However, the coefficient of determination value of $16 \%$ indicated that within-herd seroprevalence explained little of the variation in BTM ELISA ODC\%. The effect of herd size was not significant $(P=0.978$ after accounting for within-herd seroprevalence), so this variable was removed from the model.

\section{Agreement Between ELISA Versus PCR and Culture on BTM Samples}

Analysis included 192 BTM samples from the 25 study herds. Descriptive statistics of herd size, samples used per herd, and the distribution of BTM ODC\% values between the $\mathrm{PCR}$ and culture positive and negative samples are shown in Table 5. Of the BTM samples analyzed, 39\% $(\mathrm{n}=74)$ were ELISA positive, whereas only $4 \%(n=7)$ and $1 \%(n=2)$ were PCR and culture positive, respectively. Apparent prevalences differed significantly $(P<0.001)$ between ELISA and each of PCR and culture. Results matched for $61 \%$ of ELISA category and PCR results (both positive: $\mathrm{n}=$ 3 ; both negative: $\mathrm{n}=114$; ELISA positive and PCR negative: $\mathrm{n}=71$; ELISA negative and PCR positive: 


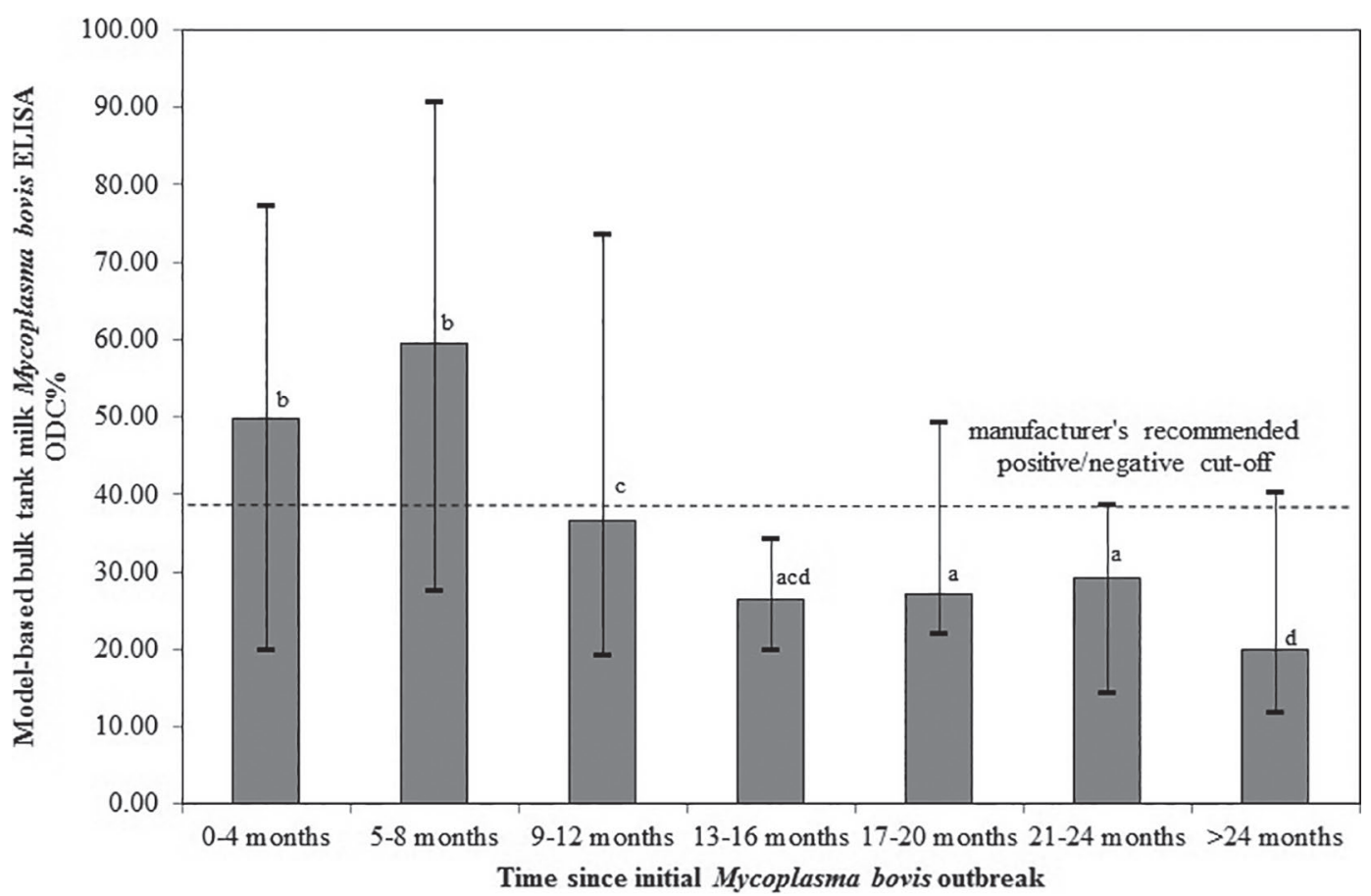

Figure 1. Model-based mean bulk tank milk Mycoplasma bovis ELISA optical density coefficient (ODC\%), with model-based minimum and maximum ODC\% for individual herds for time since initial $M$. bovis outbreak and adjusted for time since the start of the calving season. Modelbased means for time categories with different letters $(\mathrm{a}-\mathrm{d})$ are significantly different $(P<0.05)$.

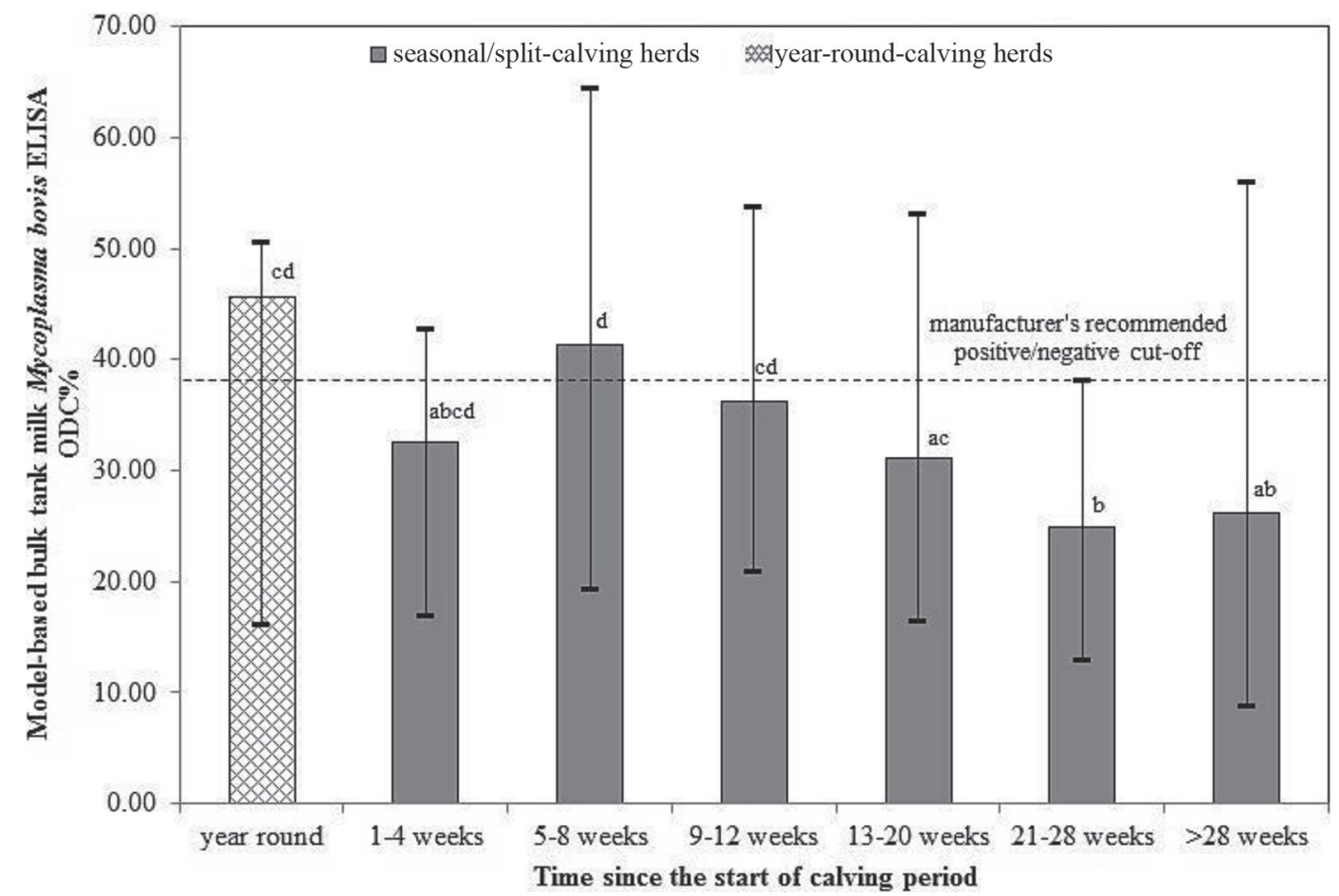

Figure 2. Model-based mean bulk tank milk ELISA optical density coefficient (ODC\%), with model-based minimum and maximum ODC\% for individual herds for time since the start of the calving period and adjusted for time since initial Mycoplasma bovis outbreak. Model-based means for calving categories with different letters $(\mathrm{a}-\mathrm{d})$ are significantly different $(P<0.05)$. 


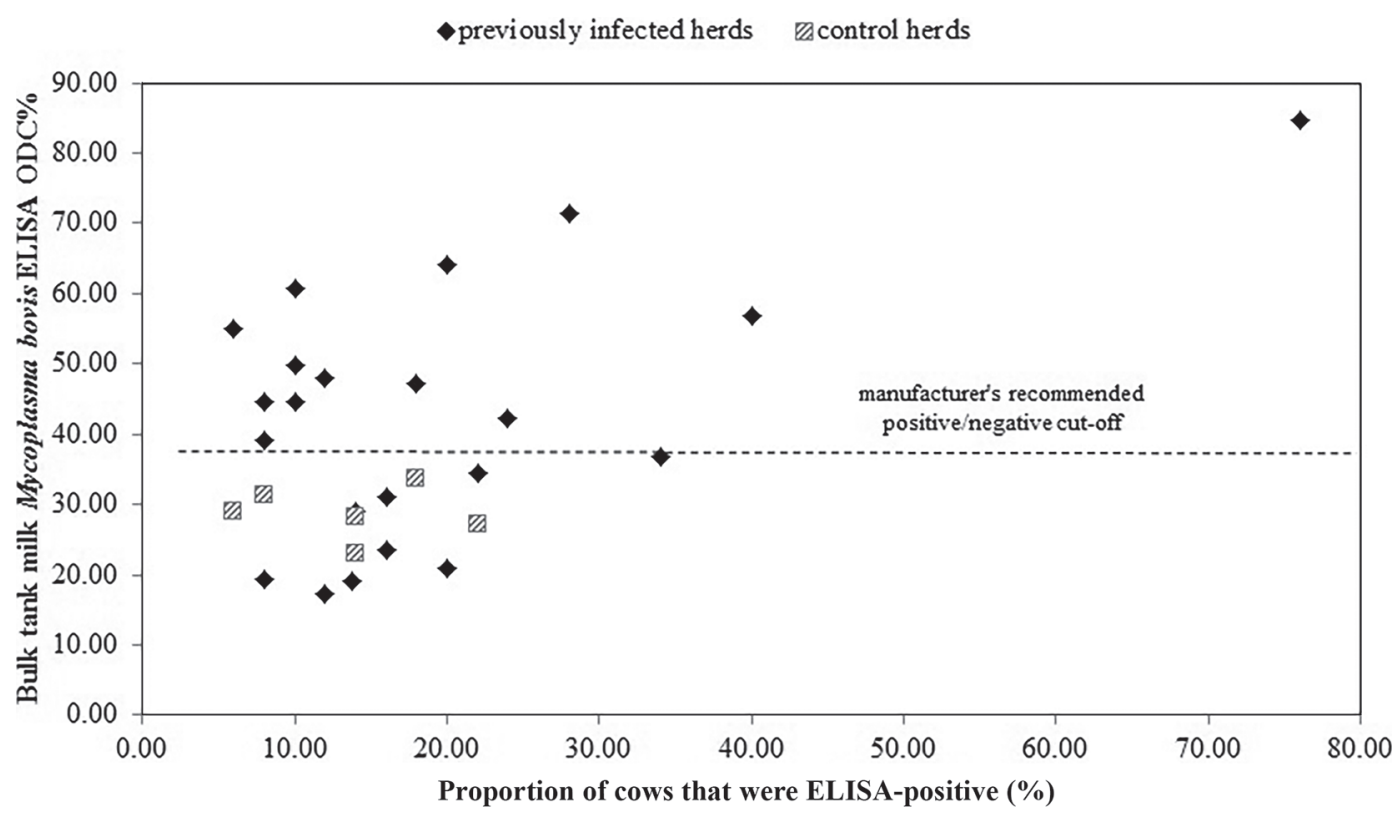

Figure 3. Scattergraph of the proportion of cows within herds exposed to Mycoplasma bovis and uninfected herds that were ELISA positive and the bulk tank milk $M$. bovis ELISA optical density coefficient (ODC\%).

$\mathrm{n}=4)$ and for $63 \%$ of ELISA category and culture results (both positive: $\mathrm{n}=2$; both negative: $\mathrm{n}=118$; ELISA positive and culture negative: $\mathrm{n}=72$; ELISA negative and culture positive: $\mathrm{n}=0$ ), and there was virtually no agreement above that expected by chance. The PABAK values were 0.22 (95\% CI: $0.08-0.36)$ and 0.25 (95\% CI: $0.11-0.39$ ) for ELISA category versus each of PCR and culture, respectively.

\section{Main BTM Versus Hospital BTM ELISA ODC\%}

Throughout the sampling period, the temporal pattern in the hospital herd BTM ELISA ODC\% generally mirrored that for the main herd but at higher ODC\% values. The mean ODC\% was significantly higher in the hospital herd compared with the main milking herd $(P<0.001)$, with means (minimum, maximum) of 54 ODC\% $(26,124)$ and 24 ODC\% $(14,43)$, respectively.

\section{DISCUSSION}

This study examined the potential use of the commercially available Bio-X Bio K 302 Mycoplasma bovis ELISA kit (Bio-X Diagnostics) as a biosecurity tool for identifying herds with past exposure to M. bovis. The BTM ODC\% was higher in year-round-calving herds and in seasonal- and split-calving herds 5 to 8 wk after the commencement of the calving period

Table 4. Descriptive statistics for the analysis of the association between the proportion of cows that were ELISA positive and the bulk tank milk (BTM) ELISA optical density coefficient (ODC\%) from 23 herds (28 samplings)

\begin{tabular}{lccc}
\hline Item & Minimum & Median & Maximum \\
\hline All herds $(\mathrm{n}=28)$ & 100 & 600 & 3,000 \\
Herd size & 6 & 14 & 76 \\
Within-herd seroprevalence of cows & 17 & 36 & 85 \\
BTM ELISA ODC\% & & & 3,000 \\
Exposed herds (n = 22) & 330 & 800 & 76 \\
Herd size & 17 & 15 & 85 \\
Within-herd seroprevalence of cows & 100 & 33 & 650 \\
BTM ELISA ODC\% & 6 & 325 & 22 \\
Control herds (n =6) & 23 & 28 & 34 \\
Herd size & & 14 & \\
Within-herd seroprevalence of cows & & & \\
BTM ELISA ODC\% & & & \\
\hline
\end{tabular}

${ }^{1}$ In Australia in 2016, the average dairy herd size was 284 cows (Dairy Australia Limited, 2016). 
and was highest sooner after $M$. bovis clinical disease outbreaks. Clinical mycoplasma disease in dairy cattle is often observed postpartum, presumably reflecting the stress of calving, and first-lactation heifers are at higher risk (Al-Abdullah and Fadl, 2006; Radaelli et al., 2011). It has been demonstrated that following exposure, seroconversion takes approximately 2 to 3 wk (Nicholas et al., 2002; Wawegama et al., 2014). Given that cows in the seasonal- and split-calving study herds calved over several weeks, it is not surprising that the model-based mean BTM ELISA ODC\% was significantly higher at 5 to 8 wk compared with $\geq 13$ wk after the commencement of the calving period in these herds (Figure 2). The subsequent decline in the model-based mean BTM ELISA ODC\% from 5 to $8 \mathrm{wk}$ onward is also consistent with studies that have demonstrated that total individual milk IgG concentrations decrease as the number of days in lactation increase (Liu et al., 2009; Eisenberg et al., 2015). Therefore, the relationship between time after the commencement of the calving period and BTM ELISA ODC\% may be attributable not just to the temporal relationship to calving but also to individual cow milk production factors. Effects of herd size on BTM ELISA ODC\% may also be affected by interactions between individual cow milk production and ELISA ODC\% values. Although herd size was included as a factor in the analysis and was found to have no significant effect, milk production data including individual cow daily milk production would be beneficial in further exploring the effects of time after the commencement of the calving period on BTM ELISA ODC\%. Interestingly, BTM samples from year-round-calving herds had a higher model-based mean BTM ELISA ODC\% compared with seasonaland split-calving herds (Figure 2), possibly due to the continued presence of recently calved cows in the lactating herd throughout the year or to differences in individual cow milk production characteristics between calving systems.

Although it has been suggested that serum M. bovis antibody concentrations remain high for several months following an outbreak (Byrne et al., 2000), little work has been conducted investigating the persistence of antibodies in milk. When looking at the model-based mean BTM ELISA ODC\% over time since the initial $M$. bovis disease outbreak, the model-based mean BTM ELISA ODC $\%$ were high at 0 to 4 mo and at 5 to 8 mo, with no significant difference between the 2 categories (Figure 1). This can be expected because seroconversion occurs approximately 2 to 3 wk after cows are challenged with M. bovis (Nicholas et al., 2002; Wawegama et al., 2014). Not surprisingly, following the observed peak at 5 to 8 mo, the model-based mean BTM ELISA ODC\% gradually decreased with time from the initial outbreak. After 12 mo, the model-based mean BTM ELISA ODC\% was less than the manufacturer's recommended cutoff of $37 \mathrm{ODC} \%$. These results suggest that the greatest chance of identifying a herd that has had past exposure to $M$. bovis occurs 0 to 8 mo after the commencement of an initial outbreak. They also indicate that, commonly, $M$. bovis antibodies can be detected in BTM for only 8 to 12 mo following an initial outbreak. Interestingly, mean BTM ELISA ODC\% was low by 12 mo after the initial outbreak despite further positive $M$. bovis culture or PCR results. Although it is possible that this poor antibody response may be attributable to antigenic shift of $M$. bovis, this seems unlikely because recent studies have demonstrated minimal genomic variability in Australian M. bovis isolates collected over a 9-yr period (Parker et al., 2016). Antigenic variation over time through phase switching (on-off) is another possible explanation. The mechanisms behind this phenomenon are not entirely understood (Bürki et al., 2015). In summary, an important antibody response in the BTM is likely to occur only when a previously unexposed herd becomes exposed. Individual cases or secondary outbreaks thereafter are unlikely to alter the BTM ODC\% substantially.

When analyzing herds with a clinical history of $M$. bovis, the present study found no significant effect of herd size on the BTM ODC\%. Petersen et al. (2016) also used a stepwise variable selection process when modeling BTM ODC\% in Danish dairy herds, including 8 herds with no clinical signs related to M. bovis in

Table 5. Descriptive statistics for analysis of the agreement between ELISA versus PCR and culture from 25 herds (192 observations), samples used per herd, and the distribution of bulk tank milk ELISA optical density coefficient (ODC\%) values between PCR and culture results

\begin{tabular}{|c|c|c|c|}
\hline Item & Minimum & Median & Maximum \\
\hline Herd size ${ }^{1}$ & 100 & 840 & 3,000 \\
\hline Samples used/herd & 1 & 6 & 32 \\
\hline ELISA ODC $\%$ for Mycoplasma bovis $\mathrm{PCR}$ positive results $(\mathrm{n}=7)$ & 18 & 26 & 56 \\
\hline ELISA ODC $\%$ for $M$. bovis PCR negative results $(\mathrm{n}=185)$ & 10 & 32 & 179 \\
\hline ELISA ODC $\%$ for $M$. bovis culture positive results $(\mathrm{n}=2)$ & 44 & 49 & 55 \\
\hline ELISA ODC $\%$ for $M$. bovis culture negative results $(\mathrm{n}=190)$ & 10 & 32 & 179 \\
\hline
\end{tabular}

${ }^{1}$ In Australia in 2016, the average dairy herd size was 284 cows (Dairy Australia Limited, 2016). 
the past 3 yr. Herd size was excluded by that process. Results from cross-sectional studies using PCR and culture have shown that although $M$. bovis can be isolated in the BTM from both small and large dairy herds, there is a positive association between herd size and detection of $M$. bovis (Fox et al., 2003; APHIS-USDA, 2008). The disparity between these results may have been attributable to the fact that all BTM samples included in the current study were obtained from herds specifically selected due to their history of $M$. bovis disease, whereas the herds included in the studies by Fox et al. (2003) and APHIS-USDA (2008) were part of a national survey and were not selected based on their $M$. bovis history. Second, most of the BTM samples included in the present study were from large herds ( $\geq 500$ in size). Third, although the likelihood of a herd being infected with $M$. bovis may be higher with increased herd size, if within-herd prevalence of exposure declines as herd size increases, the BTM ODC\% may be lowered because of the dilution effect from increased milk volume, as demonstrated in dilution series of individual positive milk samples (Vanzini et al., 2001; Tittarelli et al., 2011). Last, the outcome variables analyzed differed: the present study used continuous data in the form of BTM ODC\%, whereas Fox et al. (2003) and APHIS-USDA (2008) analyzed the presence or absence of the $M$. bovis organism.

Although BTM ODC\% was significantly associated with the proportion of cows that were ELISA positive for $M$. bovis, the coefficient of determination value was low $(16 \%)$, indicating that within-herd seroprevalence explained little of the variation in BTM ELISA ODC\%. Furthermore, several herds with positive ELISA BTM $(\geq 37$ ODC\% $)$ had within-herd seroprevalences that were lower than those of herds with negative ELISA BTM $(<37$ ODC\%). These results demonstrate that the BTM ELISA has limited utility in determining the within-herd seroprevalence of $M$. bovis exposure in Australian dairy herds. They are in contrast to those from a previous study investigating $M$. bovis (Petersen et al., 2016), where the association was closer. Petersen et al. (2016) also observed a much stronger association, with an increase of 9 ODC $\%$ per $10 \%$ increase in the proportion of cows that were ELISA positive for $M$. bovis compared with the current study, in which the estimated increase was just 1 ODC $\%$ per $10 \%$ increase. The low coefficient of determination value and weaker association in the current study may have been due, in part, to imperfect specificity of the ELISA in Australian dairy production systems. Although the specificity of this ELISA was high when used on individual serum samples from nonexposed calves (Wawegama et al., 2016), we observed seroprevalences in lactating cows varying from 6 to $22 \%$ in the 6 control herds with no known clinical history of mycoplasmosis. This may have been attributable to antigen cross-reactivity with other pathogens. For example, in humans, acute Q fever has been shown to elicit a nonspecific immunological response in some patients, including elevated IgM levels for Mycoplasma pneumoniae (Vardi et al., 2011). It is also possible that these control herds contained previously exposed animals that were causing positive serology results but that their infections had not resulted in a clinical outbreak. The absence of a close association between BTM ODC\% and the proportion of cows that were ELISA positive for $M$. bovis may also have been attributable to sampling occurring farther away from the herds' clinical outbreaks. Further, with the exception of a few outlier herd samplings with high within-herd seroprevalences, the range of within-herd seroprevalence results was quite narrow compared with the range of BTM ODC\% (Figure 3), a similar finding to a Mycobacterium avium ssp. paratuberculosis ELISA (Nielsen and Toft, 2014). The association may be closer in populations with more variation in the proportion of cows that are ELISA positive. Finally, the absence of a close association may have been partly attributable to poor sensitivity of the Bio-X ELISA at the individual animal level. Sensitivity of this ELISA has been estimated as 37\% (95\% CI: 22-54) relative to an IgG ELISA in sera from individual animals (Wawegama et al., 2016) and 60\% (95\% posterior credibility interval: 38-96) in BTM (Nielsen et al., 2015). However, although not all herds with a known history of $M$. bovis disease had a positive BTM $(\geq 37$ ODC $\%)$, the BTM ELISA may be more useful in identifying absence or presence of previous exposure to $M$. bovis at the herd level. Given the reported relative poor sensitivity of the assay (Nielsen et al., 2015; Wawegama et al., 2016), to increase the chance of identifying a previously exposed herd, when possible, sampling in seasonal- and splitcalving herds should be targeted to 5 to 8 wk after the start of the calving period - the period where mean BTM ODC\% was highest. If the BTM ELISA is to be effective as a biosecurity tool for avoiding purchase of cattle infected with $M$. bovis, negative BTM ELISA results must indicate that the probability that $M$. bovis is present in the vendor's herd is very low (i.e., the negative predictive value must be very high). In any randomly selected Australian dairy herd, the probability that $M$. bovis is present is currently low because the herd-level prevalence of $M$. bovis in Australia is low (Morton et al., 2014). This probability would be slightly lower in herds with no clinical history suspicious of $M$. bovis, and negative BTM ELISA results from these herds would indicate that the probability that $M$. bovis is present is even lower, even if the sensitivity of the BTM ELISA is only modest. Although the reduction 
in probability between such herds without and with negative BTM ELISA results would generally be small, given the serious consequences of introducing $M$. bovis into unexposed (and hence immunologically naïve) herds, this additional information may be valuable.

A much higher percentage of BTM samples were ELISA positive (39\%) than were PCR (4\%) or culture (1\%) positive, resulting in low PABAK values of 0.22 (95\% CI: $0.08-0.36)$ and 0.25 (95\% CI: 0.11-0.39) for ELISA category versus each of PCR and culture, respectively. This poor agreement can be expected given that ELISA measures antibodies for past exposure, whereas PCR and culture measure the presence of the pathogen. The study by Nielsen et al. (2015) also found that a high proportion of BTM samples were positive by ELISA but negative by PCR. This has also been demonstrated with individual animals (Byrne et al., 2000; Akan et al., 2014). Assuming that the specificity of the ELISA is at least modest, this indicates that past $M$. bovis exposure may be far more common than shedding of the pathogen. If so, the presence of both bacterial DNA and antibodies in the BTM sample at the same time is unlikely, particularly given that clinically affected animals, which are most likely to be shedding the pathogen, are often in a separate hospital herd and may or may not have developed an immune response to a new infection. For this reason, the use of the BTM ELISA as a biosecurity tool for identifying herds that have been exposed to $M$. bovis may be of value in providing additional information from a different biological perspective and may complement the use of PCR or culture in identifying higher risk herds from which animals are being considered for purchase.

In the study by Petersen et al. (2016), although there was an association between the within-herd apparent prevalence of the main milking herd and the BTM ODC\% results, this was not the case for the within-herd seroprevalence of young stock (0-12 mo of age). This would be expected because the BTM ODC\% is reflective only of the animals that are contributing milk to the BTM sample. However, when considering the purchase of nonlactating animals (e.g., heifers) that were bred in the herd, the BTM ELISA ODC\% would provide information about their herd of origin. Milk from lactating animals in the hospital herd is also excluded from this sample type because the status of these animals is not reflected in the main herd BTM ODC\%. This is an important point because a recommended strategy for controlling transmission of Mycoplasma spp. within a herd is to identify and segregate infected animals into a separate herd (Fox et al., 2005). Therefore, currently infected animals are often not contributing to the main herd BTM. This may result in negative main herd BTM PCR and culture results despite cows with Mycoplasma clinical disease having been recently removed from that herd. Therefore, sampling the hospital herd BTM may be beneficial in addition to sampling the main herd BTM to assess the joint status of both herds. When comparing BTM samples from the main herd and the hospital herd on one farm with a history of $M$. bovis, the mean BTM ELISA ODC\% for the hospital herd was significantly higher. When comparing patterns over time, the hospital herd BTM ELISA ODC\% generally mirrored that of the main herd, however at higher concentrations. Although the main herd BTM ELISA ODC\% rarely increased above the recommended cutoff to be considered positive, the hospital herd was positive at most sampling points. This may be attributable to a higher chance of animals exposed to $M$. bovis being in the hospital herd compared with the main herd due to disease management strategies. Although these results are based only on a single herd and therefore should be interpreted with caution, we suggest that hospital herd BTM samples may be more effective than main herd BTM samples in identifying herds with a history of $M$. bovis. However, we recommend collecting both hospital herd and main herd BTM samples because hospital herd BTM may practically be a more difficult sample to obtain. These results also suggest that, from a disease management point of view, the hospital herd BTM sample may be useful for internal monitoring of $M$. bovis activity within a herd. Additional work is required to further explore the role of hospital herd BTM sampling.

If BTM ELISA is to be used as a biosecurity tool to screen herds from which animals are being considered for purchase, along with high sensitivity, high specificity is desirable to minimize the number of follow-up investigations required in vendors' herds due to falsepositive results. All 6 control herds were negative $(<37$ ODC\%). These results, coupled with an estimated BTM specificity of $97.3 \%$ (95\% posterior credibility interval: 94.0-99.8) by Nielsen et al. (2015), are suggestive of at least modest specificity at the herd level when used on BTM samples. However, given that all 6 control herds included in this study were from one state and that all used the year-round calving system, further sampling of control herds expanding to other regions and calving systems is required to assess specificity of the BTM ELISA. However, these observations in our control herds are consistent with findings by Petersen et al. (2016), who, with the exception of a control herd that experienced a clinical outbreak during the trial, collected few positive ELISA BTM samples from 7 control herds with no known clinical signs of $M$. bovis in the past $3 \mathrm{yr}$ (Mette B. Petersen, University of Copenhagen, Frederiksberg, Denmark, personal communication). However, it is not known whether the herds 
included in the control group by Petersen et al. (2016) had a clinical history of $M$. bovis disease greater than 3 yr ago or whether they were subclinically infected. In the current study we selected 6 herds with no clinical signs of $M$. bovis disease within the last $5 \mathrm{yr}$ as control herds, and due to the long-standing close association with the University of Sydney LVTRU, it is likely that all of these herds had no clinical signs of $M$. bovis disease for many years.

\section{CONCLUSIONS}

This study was conducted to evaluate the use of the commercially available Bio-X Bio K 302 Mycoplasma bovis ELISA kit (Bio-X Diagnostics) on BTM samples as a biosecurity tool for identifying herds that have past exposure to $M$. bovis and, as such, pose a biosecurity risk to unexposed herds planning to purchase and introduce new stock. Following an initial outbreak of $M$. bovis, the greatest window of opportunity for identifying herds with past exposure via ELISA was 0 to 8 mo postoutbreak. In seasonal- and split-calving herds, significantly higher model-based mean BTM ELISA ODC\% results were observed 5 to 8 wk following the commencement of the calving season, presumably because of the effects of calving stress. Although the BTM ELISA ODC\% was significantly associated with the within-herd seroprevalence, the BTM ELISA had limited utility in determining the within-herd seroprevalence of $M$. bovis exposure in study herds. A significant difference in results was obtained from the BTM ELISA assay and PCR or culture. A much higher percentage of BTM samples were ELISA positive, and there was virtually no agreement between ELISA category and either PCR or culture other than that expected by chance. Finally, the hospital herd BTM ELISA ODC\% mirrored that of the main herd BTM but at higher concentrations. Therefore, the hospital herd BTM may be a more effective sample type for detecting herds with past exposure to $M$. bovis. Although further work is needed in investigating the herd-level specificity of this assay, this study demonstrates that the use of the BTM ELISA may be complementary to the use of BTM PCR or culture as a prepurchase herd screening tool to mitigate the risk of purchasing infected stock.

\section{ACKNOWLEDGMENTS}

The authors acknowledge Dairy Australia (Southbank, Victoria, Australia) for their financial support. The authors also gratefully thank all the veterinary practitioners and dairy producers throughout Australia who assisted with sample collection and made their animals available. The technical assistance provided by Ann-Marie House and Karen Mathews (Sydney School of Veterinary Science, The University of Sydney, Australia) is greatly appreciated. Statistical advice provided by Evelyn Hall (University of Sydney) has also been invaluable. The authors declare that no conflicts of interest exist.

\section{REFERENCES}

Akan, M., O. Babacan, E. Torun, H. K. Mustak, and T. Oncel. 2014 Diagnosis of Mycoplasma bovis infection in cattle by ELISA and PCR. Kafkas Univ. Vet. Fak. 20:249-252.

Al-Abdullah, H. A., and E. A. Fadl. 2006. Mycoplasma mastitis in dairy cattle herds in Saudi Arabia. Vet. Rec. 159:88-89.

USDA APHIS (Animal and Plant Health Inspection Service). 2008. Prevalence of contagious mastitis pathogens on US dairy operations, 2007. Info sheet. USDA APHIS, Fort Collins, CO.

Biddle, M. K., L. K. Fox, and D. D. Hancock. 2003. Patterns of mycoplasma shedding in the milk of dairy cows with intramammary mycoplasma infection. J. Am. Vet. Med. Assoc. 223:1163-1166.

Bürki, S., J. Frey, and P. Pilo. 2015. Virulence, persistence and dissemination of Mycoplasma bovis. Vet. Microbiol. 179:15-22.

Byrne, W. J., H. J. Ball, N. Brice, R. McCormack, S. E. Baker, R. D. Ayling, and R. A. J. Nicholas. 2000. Application of an indirect ELISA to milk samples to identify cows with Mycoplasma bovis mastitis. Vet. Rec. 146:368-369.

Cottew, G. S. 1970. Mycoplasmas isolated from cattle in Australia. Aust. Vet. J. 46:378-381.

Dairy Australia Limited. 2014. Mycoplasma in dairy herds. Fact sheet. Accessed May 12, 2016. http://www.dairyaustralia.com.au/ / media/Documents/Animal\%20management/Mastitis/Countdown $\%$ 20news $/ 2016 \% 20$-\% 20Mycoplasma \%20in \%20dairy $\% 20$ herds $\% 20$ fact $\% 20$ sheet.pdf.

Dairy Australia Limited. 2016. Dairy cows and farms. Accessed May 12, 2017. http://www.dairyaustralia.com.au/Markets-and -statistics/Farm-facts/Cows-and-Farms.aspx.

Eisenberg, S. W., E. Veldman, V. P. Rutten, and A. P. Koets. 2015. A longitudinal study of factors influencing the result of a Mycobacterium avium ssp. paratuberculosis antibody ELISA in milk of dairy cows. J. Dairy Sci. 98:2345-2355.

Fox, L. K., D. D. Hancock, A. Mickelson, and A. Britten. 2003. Bulk tank milk analysis: Factors associated with appearance of $\mathrm{Myco-}$ plasma sp. in milk. J. Vet. Med. B Infect. Dis. Vet. Public Health $50: 235-240$.

Fox, L. K., J. H. Kirk, and A. Britten. 2005. Mycoplasma mastitis: A review of transmission and control. J. Vet. Med. B Infect. Dis. Vet. Public Health 52:153-160.

Higuchi, H., H. Iwano, S. Gondaira, K. Kawai, and H. Nagahata. 2011. Prevalence of Mycoplasma species in bulk tank milk in Japan. Vet. Rec. 169:442.

Justice-Allen, A., J. Trujillo, G. Goodell, and D. Wilson. 2011. Detection of multiple Mycoplasma species in bulk tank milk samples using real-time PCR and conventional culture and comparison of test sensitivities. J. Dairy Sci. 94:3411-3419.

Liu, G. L., J. Q. Wang, D. P. Bu, J. B. Cheng, C. G. Zhang, H. Y. Wei, Z. F. Zhou, L. Y. Zhou, H. Hu, and X. L. Dong. 2009. Factors affecting the transfer of immunoglobulin G1 into the milk of Holstein cows. Vet. J. 182:79-85.

Maunsell, F. P., A. R. Woolums, D. Francoz, R. F. Rosenbusch, D. L. Step, D. J. Wilson, and E. D. Janzen. 2011. Mycoplasma bovis infections in cattle. J. Vet. Intern. Med. 25:772-783.

Morton, J., J. Malmo, J. House, G. Mein, M. Izzo, and J. Penry. 2014. Mycoplasma bovis in Australian dairy herds. Aust. Vet. J. 92:322-323.

Nicholas, R. A. 2011. Bovine mycoplasmosis: Silent and deadly. Vet. Rec. 168:459-462.

Nicholas, R. A., R. D. Ayling, and L. P. Stipkovits. 2002. An experimental vaccine for calf pneumonia caused by Mycoplasma bovis: 
Clinical, cultural, serological and pathological findings. Vaccine 20:3569-3575.

Nielsen, P. K., M. B. Petersen, L. R. Nielsen, T. Halasa, and N. Toft. 2015. Latent class analysis of bulk tank milk PCR and ELISA testing for herd level diagnosis of Mycoplasma bovis. Prev. Vet. Med. 121:338-342.

Nielsen, S. S., and N. Toft. 2014. Bulk tank milk ELISA for detection of antibodies to Mycobacterium avium ssp. paratuberculosis: Correlation between repeated tests and within-herd antibody-prevalence. Prev. Vet. Med. 113:96-102.

Parker, A. M., J. K. House, M. S. Hazelton, K. L. Bosward, and P. A Sheehy. 2017. Comparison of culture and a multiplex probe PCR for identifying Mycoplasma species in bovine milk, semen and swab samples. PLoS One 12:e0173422.

Parker, A. M., A. Shukla, J. K. House, M. S. Hazelton, K. L. Bosward, B. Kokotovic, and P. A. Sheehy. 2016. Genetic characterization of Australian Mycoplasma bovis isolates through whole genome sequencing analysis. Vet. Microbiol. 196:118-125.

Petersen, M. B., K. Krogh, and L. R. Nielsen. 2016. Factors associated with variation in bulk tank milk Mycoplasma bovis antibodyELISA results in dairy herds. J. Dairy Sci. 99:3815-3823.

Radaelli, E., V. Castiglioni, M. Losa, V. Benedetti, R. Piccinini, R. A. Nicholas, E. Scanziani, and M. Luini. 2011. Outbreak of bovine clinical mastitis caused by Mycoplasma bovis in a North Italian herd. Res. Vet. Sci. 91:251-253.

Tittarelli, M., B. Bonfini, F. De Massis, A. Giovannini, and M. Scacchia. 2011. Standardisation of an indirect enzyme-linked immunosorbent assay for the detection of Brucella antibodies in milk from water buffalo (Bubalus bubalis) in Italy. Vet. Ital. 47:59-64.

Vanzini, V. R., N. R. Aguirre, B. S. Valentini, S. T. de Echaide, C. I. Lugaresi, M. D. Marchesino, and K. Nielsen. 2001. Comparison of an indirect ELISA with the Brucella milk ring test for detection of antibodies to Brucella abortus in bulk milk samples. Vet. Microbiol. 82:55-60.

Vardi, M., N. Petersil, A. Keysary, S. Rzotkiewicz, A. Laor, and H. Bitterman. 2011. Immunological arousal during acute Q fever infection. Eur. J. Clin. Microbiol. Infect. Dis. 30:1527-1530.

Wawegama, N. K., G. F. Browning, A. Kanci, M. S. Marenda, and P. F. Markham. 2014. Development of a recombinant protein-based enzyme-linked immunosorbent assay for diagnosis of Mycoplasma bovis infection in cattle. Clin. Vaccine Immunol. 21:196-202.

Wawegama, N. K., P. F. Markham, A. Kanci, M. Schibrowski, S. Oswin, T. S. Barnes, S. M. Firestone, T. J. Mahony, and G. F. Browning. 2016. Evaluation of an IgG enzyme-linked immunosorbent assay as a serological assay for detection of Mycoplasma bovis infection in feedlot cattle. J. Clin. Microbiol. 54:1269-1275. 